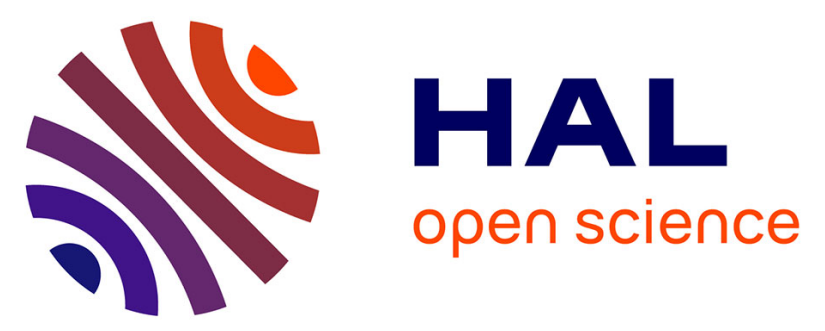

\title{
Description of transport mechanisms in a very long wave infrared quantum cascade detector under strong magnetic field
}

François-Régis Jasnot, Simon Maëro, Nicolas Péré-Laperne, Louis-Anne de Vaulchier, Yves Guldner, Francesca Carosella, Robson Ferreira, Alexandre Delga, Laetitia Doyennette, Vincent Berger, et al.

\section{To cite this version:}

François-Régis Jasnot, Simon Maëro, Nicolas Péré-Laperne, Louis-Anne de Vaulchier, Yves Guldner, et al.. Description of transport mechanisms in a very long wave infrared quantum cascade detector under strong magnetic field. Journal of Applied Physics, 2012, pp.123705. hal-00911467

\section{HAL Id: hal-00911467 \\ https://hal.science/hal-00911467}

Submitted on 4 Dec 2013

HAL is a multi-disciplinary open access archive for the deposit and dissemination of scientific research documents, whether they are published or not. The documents may come from teaching and research institutions in France or abroad, or from public or private research centers.
L'archive ouverte pluridisciplinaire HAL, est destinée au dépôt et à la diffusion de documents scientifiques de niveau recherche, publiés ou non, émanant des établissements d'enseignement et de recherche français ou étrangers, des laboratoires publics ou privés. 


\title{
Description of transport mechanisms in a very long wave infrared quantum cascade detector under strong magnetic field
}

\author{
François-Régis Jasnot, ${ }^{1}$ Simon Maëro, ${ }^{1}$ Nicolas Péré-Laperne, ${ }^{1,} *$ Louis-Anne de Vaulchier, ${ }^{1, \dagger}$ Yves Guldner, ${ }^{1}$ Francesca \\ Carosella, ${ }^{1}$ Robson Ferreira, ${ }^{1}$ Alexandre Delga, ${ }^{2}$ Laetitia Doyennette, ${ }^{2}$ Vincent Berger, ${ }^{2}$ Virginie Trinité, ${ }^{3}$ and Mathieu Carras ${ }^{3}$ \\ ${ }^{1}$ Laboratoire Pierre Aigrain, École Normale Supérieure, CNRS (UMR 8551), \\ Université P. et M. Curie, Université Paris Diderot, 24 rue Lhomond, 75231 Paris Cedex 05, France \\ ${ }^{2}$ Laboratoire Matériaux et Phénomènes Quantiques, Université Paris Diderot - Paris 7, \\ CNRS - UMR7162, Bâtiment Condorcet, 75205 Paris Cedex 13, France \\ ${ }^{3}$ Alcatel-Thales 3-5 lab, Route départementale 128, 91767 Palaiseau Cedex, France
}

\begin{abstract}
Measurements of current have been performed on a very long wave infrared quantum cascade detector under strong magnetic field applied parallel to the growth axis, both under dark and light conditions. The analysis of dark current as a function of temperature highlights three regimes of transport involving the different energy levels of the structure. For photocurrent analysis, we developed a model based on a rate equation approach taking into account all the electronic levels of the structure. This model is in agreement with the oscillatory component of the experimental magnetophotocurrent. It allows to identify the key points controlling the electronic transport such as extraction from the upper level of the optically active quantum well, location of ionized impurities and scattering mechanisms involved in the structure. This work is valuable for the future conception of highperformance quantum cascade detectors in infrared and far infrared range.
\end{abstract}

PACS numbers: 71.70.Di, 72.10.-d, 73.23.-b, 73.43.Qt, 75.47.-m

\section{INTRODUCTION}

Quantum cascade detectors ${ }^{1}$ (QCDs) have been proposed for application in a wide domain of energy radiation extending from near infrared ${ }^{2}$ to terahertz ${ }^{3,4}$ through the long wave ${ }^{5,6}(8$ $-12 \mu \mathrm{m})$ and more recently very long wave ${ }^{7,8}(12-20 \mu \mathrm{m})$ infrared (VLWIR). VLWIR is a spectral range of high interest because of numerous applications, ${ }^{7,9-12}$ e.g. meteorology (global atmospheric temperature profile, relative humidity profile, cloud characteristics and atmospheric chemistry) or astronomy (analysis of molecular clouds and star forming regions of our galaxy, spectroscopy of molecular hydrogen, detection of faint objects such as exoplanets or protoplanetary dust disks). Quantum well infrared photodetectors (QWIPs) have been proposed for VLWIR detection and look very promising. ${ }^{9,10}$ However, in photoconductive mode, they operate under an applied bias leading to a strong dark current that saturates the read-out integrated circuit capacity and a significant noise level. In contrast, QCDs are working in a photovoltaic mode, under no applied electric field, making them good candidates for higher working temperature, longer integration times and lower dark current.. They are constituted of several periods, each one composed of two main parts. The first one is the "active region" where absorption of infrared photons occurs. The second one is the "cascade" transferring electrons from one period to the other by scattering processes. $^{1,5}$

The VLWIR QCD studied in this paper was first presented in Ref. 8. The maximum spectral responsivity was predicted at a negative bias, $V=-0.6 \mathrm{~V}$, but experiment has revealed that maximal value was reached at $V=-1.1 \mathrm{~V}$. This highlights the difficulty of modelling and understanding of this complex structure. In this context, the application of a magnetic field is a valuable experimental technique to evaluate the different contributions of scattering mechanisms involved in these quantum cascade structures. ${ }^{4,13-18}$ The analysis is based on the Landau quantization. Besides, study of QCDs under magnetic field is of high interest for the comprehension of quantum well structures properties. Indeed, in contrast with other detectors such as QWIPs where transport involves both two dimensional (2D) and 3D electronic states in the quantum well and in the continuum, respectively, the transport in QCDs is fully $2 \mathrm{D}$ and a complete modelling of the device is possible down from quantum principles. For example, the different electronic paths contributing to the dark current in QCDs can be highlighted thanks to magnetotransport. ${ }^{19}$ Under illumination, magnetomeasurements of photocurrent in a $8 \mu \mathrm{m}$ QCD have evidenced the crucial role of ionized impurities in QCD transport properties. ${ }^{20}$

In this paper, after describing the QCD structure in Sec. II, we focus on the analysis of dark current as a function of temperature in Sec. III. It is the first step to characterize the transport properties of this QCD. The second step, in Sec. IV, is to analyse the perturbation of this dark current due to photogenerated carriers, namely study QCD under illumination. Section $\mathrm{V}$ is devoted to the description of the model which is used in Sec. VI to analyse experimental data.

\section{QCD STRUCTURE}

The QCD studied in this paper is a GaAs/AlGaAs heterostructure for VLWIR detection. Its detection wavelength is $14.3 \mu \mathrm{m} .{ }^{8}$ The sample consists of 30 identical periods of four coupled GaAs quantum wells (QWs). Figure 1 shows one period of the QCD structure. Barriers are made of $\mathrm{Al}_{0.27} \mathrm{Ga}_{0.73} \mathrm{As}$ in order to reach a conduction band offset of $V_{0}=222 \mathrm{meV}$. Starting from the first QW, the layer sequence in $\AA$ is as follows $99 / \mathbf{6 0} / 37 / \mathbf{4 1} / 47.5 / \mathbf{5 0} / 50 / \mathbf{5 8}$ (the barrier widths are represented in bold types). The thickest QW of each period is $n$-doped $\left(N_{\mathrm{e}}=2.2 \times 10^{11} \mathrm{~cm}^{-2}\right)$ to populate 


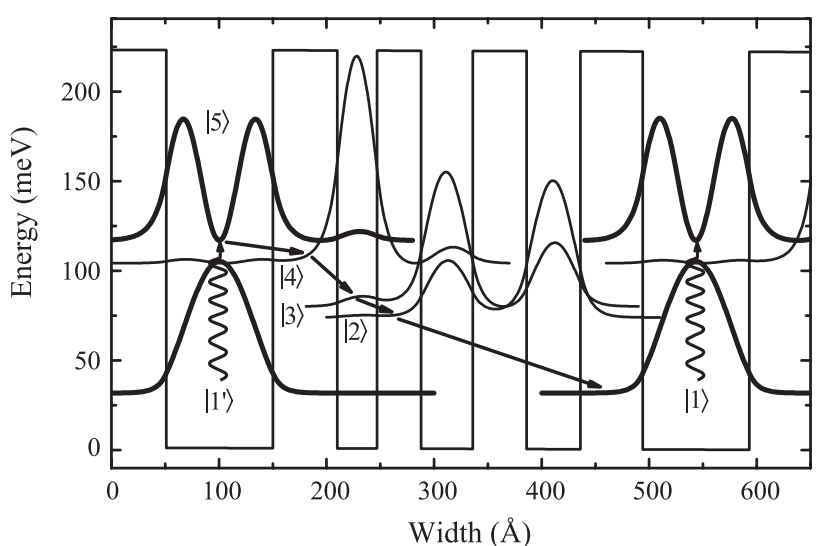

FIG. 1: Conduction band diagram of one period of a $14.3 \mu \mathrm{m}$ QCD for VLWIR detection under zero applied bias showing the square moduli of the wave function of each energy level $|i\rangle$. Waved arrows represent photon absorption and straight arrows illustrate the electronic path in the cascade after a detection event.

the first energy level $\left|1^{\prime}\right\rangle$ in the conduction band with electrons (see Fig. 1). Contrary to conventional quantum cascade structures like quantum cascade lasers, ${ }^{21}$ the doping level is much higher and dopants are placed in the optically active QW, i.e. in the active region of the QCD. The performances of this QCD are detailed in Ref. 8.

As illustrated on Fig. 1, under illumination, electrons localized on the lowest energy level of the structure $\left|1^{\prime}\right\rangle$ are photoexcited to the upper level $|5\rangle$. They are then transferred through scattering events to the QWs of the cascade where levels are labelled $|i\rangle$ with $i \in\{1, \ldots, 4\}$. By closing the circuit, a significant photocurrent is expected without any applied bias. The studied sample is constituted of square-shaped mesas with $100 \mu \mathrm{m}$ side length obtained by reactive ion etching.

\section{DARK CURRENT}

Under dark conditions, at zero applied voltage, the QCD is at equilibrium, resulting in zero current. ${ }^{19}$ All the intersubband transitions verify micro-reversibility: the contrapropagating fluxes are equal. They nonetheless generate noise, ${ }^{22,23}$ and their study is crucial to understand the detector performances. Once a bias is applied, the micro-reversibility condition is broken and dark current is generated. The objective in this section is to analyse the relative contributions of the intercascade transitions involved in the dark current as a function of the temperature in a QCD.

The QCD is mounted in a helium cooled cryostat inside an insert at the center of a superconducting coil capable of producing fields up to $15 \mathrm{~T}$. The magnetic field lines are perpendicular to the plane of the quantum wells, i.e. parallel to the current flow direction. The experiment consists in measuring the dark current $\left(I_{\text {dark }}\right)$ along the device, the magnetic field being swept from zero to its maximum value, at seven different temperatures: 4.5, 20, 40, 60, 80, 100 and $120 \mathrm{~K}$.

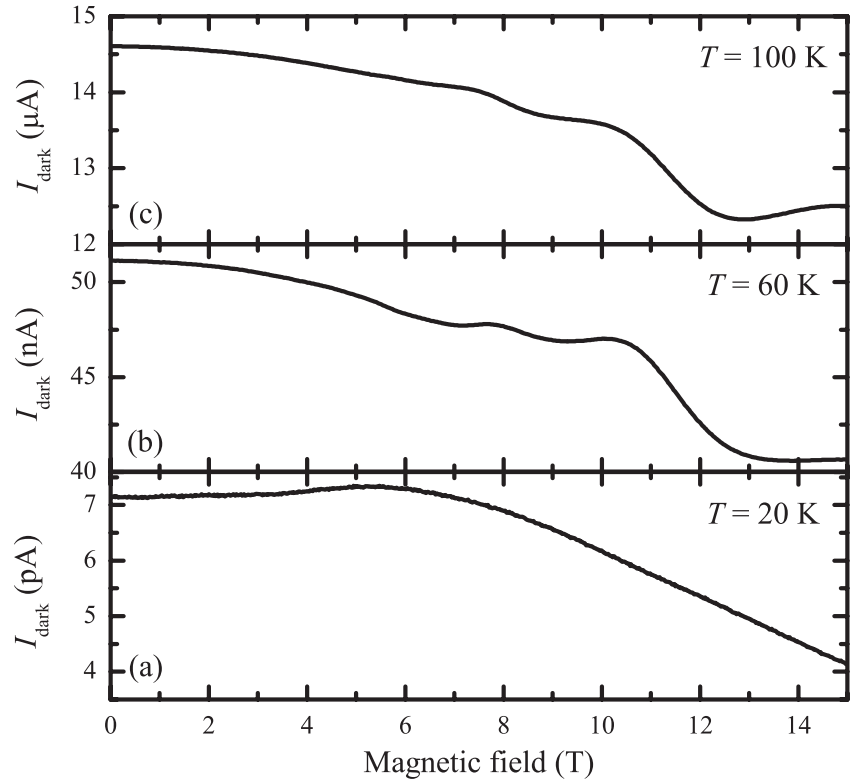

FIG. 2: Dark current as a function of magnetic field for a fixed value of the voltage $V=-0.1 \mathrm{~V}$ at (a) $20 \mathrm{~K}$, (b) $60 \mathrm{~K}$ and (c) $100 \mathrm{~K}$.

Current measurements have been performed under a low bias of $V=-0.1 \mathrm{~V}$ for all temperatures. Typical behaviours of $I_{\text {dark }}$ as a function of magnetic field are shown in Fig. 2 for three temperatures: $20 \mathrm{~K}$ (a), $60 \mathrm{~K}$ (b) and $100 \mathrm{~K}$ (c). The dark current exhibits clear oscillations as a function of $B$ superimposed on a continuous quadratic decreasing background which is attributed to the magnetoresistance of the contacts of the sample. ${ }^{24}$ Position and intensity of minima and maxima strongly depend on the temperature. Finally, note that the measured current varies by roughly 7 orders of magnitude from 4.5 up to $120 \mathrm{~K}$.

At equilibrium, cascades are well described as thermalised. ${ }^{25}$ Levels $|1\rangle$ and $\left|1^{\prime}\right\rangle$ having the smallest diffusion rates, they contain almost all the electrons. Thus, critical transitions governing the dark current are the LO-phonon assisted parallel diffusions between level $\left|1^{\prime}\right\rangle$ and the neighbouring cascade. Only electron-LO-phonon absorption has been taken into account, because it is the predominant mechanism in GaAs QCD dark current. ${ }^{25,26}$ Indeed the differences of energy involved for intercascade transitions are higher than $\hbar \omega_{\mathrm{LO}}$, the LO-phonon energy. Thus other scattering mechanisms can be neglected. The application of a magnetic field perpendicular to the layers of the QCD breaks the two-dimensional parabolic energy dispersion of each subband $E_{i}(\mathbf{k})$ into a ladder of discrete Landau Levels (LLs) $|i, j\rangle$ with an energy

$$
E_{i, j}=E_{i}^{0}+\left(j+\frac{1}{2}\right) \hbar \omega_{\mathrm{c}},
$$

and separated by the cyclotron energy $\hbar \omega_{\mathrm{c}}=\hbar e B / \mathrm{m}^{*}$, where $i$ is the subband index, $E_{i}^{0}$ the energy of the subband edge at zero magnetic field, $j$ the LL integer index, $B$ the magnetic field, and $m^{*}$ the energy dependent electron effective mass. The effect of $B$ on $I_{\text {dark }}$ is quite similar to that in active region of a quantum cascade structure: depending on the value of 


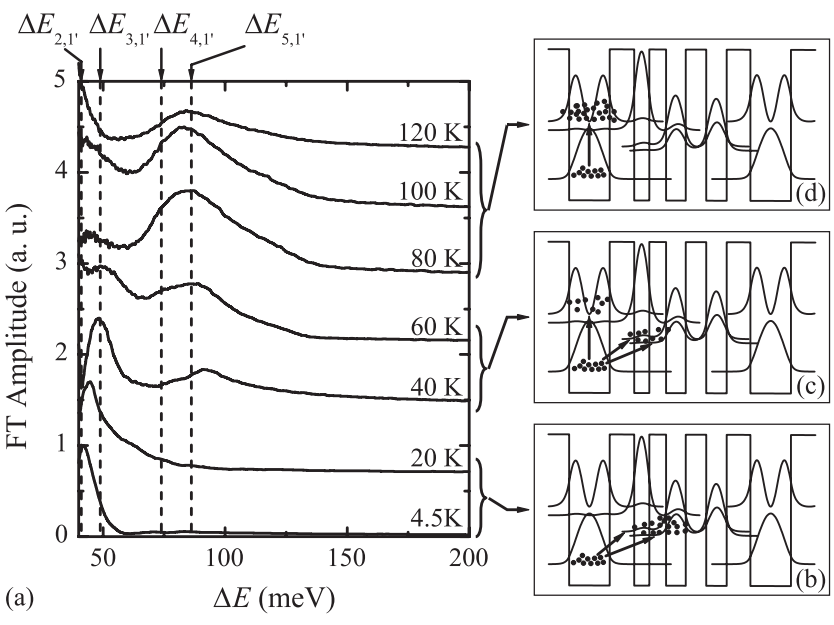

FIG. 3: (a) Fourier transform amplitude of the oscillatory part of $I_{\text {dark }}$ vs. $\Delta E=\hbar e B / m^{*}+\hbar \omega_{\mathrm{LO}}$ for $4.5,20,40,60,80,100$ and $120 \mathrm{~K}$. $\Delta E_{i, 1^{\prime}}$ are reported in vertical dashed line. (b,c,d) Guides for the eyes to identify the electronic transitions observed at (b) $4.5 \& 20 \mathrm{~K}$, (c) $40 \& 60 \mathrm{~K}$ and (d) $80,100 \& 120 \mathrm{~K}$. Electrons are symbolised by small points.

$B$, the LL arrangement strongly influences the absorption or emission of LO-phonon. ${ }^{17,19}$

The analysis of experimental data has been realized following the procedure used in Ref. 19, based on the Fourier transform (FT) of the oscillatory part of $I_{\text {dark }}$. This method is an efficient way to reveal the different transitions involved in the dark current. The FT of the dark current measurements for all temperatures are reported on Fig. 3(a). Their amplitudes have been plotted as a function of $\Delta E=\hbar e B / m^{*}+\hbar \omega_{\mathrm{LO}}$. This allows a clear identification of the energy transitions between level $\left|1^{\prime}\right\rangle$ and $|i\rangle: \Delta E_{i, 1^{\prime}}$. The different values of $\Delta E_{i, 1^{\prime}}$ have been extracted from the calculated band structure at $V=-0.1 \mathrm{~V}$ and have been reported on Fig. 3(a). Three regimes of temperature can be identified. The first, the second and the third one, contain respectively the curves at 4.5 and $20 \mathrm{~K}$, those at 40 and $60 \mathrm{~K}$ and these at 80,100 and $120 \mathrm{~K}$. In the curves at 4.5 and $20 \mathrm{~K}$, one peak is identified at low energy, around $45 \mathrm{meV}$, and is attributed to the contribution of transitions $\left|1^{\prime}\right\rangle \rightarrow|2\rangle$ and $\left|1^{\prime}\right\rangle \rightarrow|3\rangle$. In the curves at 40 and $60 \mathrm{~K}$, this peak decreases while at the same time a broad peak emerges at higher energy, around $80 \mathrm{meV}$ corresponding to transitions $\left|1^{\prime}\right\rangle \rightarrow|4\rangle$ and $\left|1^{\prime}\right\rangle \rightarrow|5\rangle$. Finally, at 80, 100 and $120 \mathrm{~K}$ this behaviour is reinforced. The peak at lower energy disappears and the one at higher energy translates to $85 \mathrm{meV}$ which corresponds to the transition $\left|1^{\prime}\right\rangle \rightarrow|5\rangle$. In addition, a small wide peak appears at low energy. It is attributed to the intracascade transitions and/or elastic scattering. ${ }^{19}$

As a further proof for this behaviour, we calculate, following Ref. 19 and 27, the LO-phonon absorption scattering rates $\Gamma_{1^{\prime}, i}^{\mathrm{LO} \text { phonon-a }}$, i.e. the number of transitions per second from level $\left|1^{\prime}\right\rangle$ to level $|i\rangle$ with $i \in\{2, \ldots, 5\}$ thanks to LO-phonon absorption for an applied voltage $V=-0.1 \mathrm{~V}$. The results are provided in Table I for the three regimes, at 20, 60 and
TABLE I: Values of the LO-phonon absorption scattering rates $\Gamma_{1^{\prime}, i}^{\mathrm{LO} \text { phonon-a }}$ for $i \in\{2, \ldots, 5\}$ in $\mathrm{s}^{-1}$ at 20,60 and $100 \mathrm{~K}$ for an applied voltage $V=-0.1 \mathrm{~V}$. Bold characters correspond to the highest values of the global transition rates for each temperature.

\begin{tabular}{lccc}
\hline \hline$\Gamma_{1^{\prime}, i}^{\text {LO phonon-a }}$ & $20 \mathrm{~K}$ & $60 \mathrm{~K}$ & $100 \mathrm{~K}$ \\
\hline$\left|1^{\prime}\right\rangle \rightarrow|2\rangle$ & $\mathbf{4 . 1} \times \mathbf{1 0}^{-\mathbf{3}}$ & $\mathbf{2 . 2} \times \mathbf{1 0}^{\mathbf{4}}$ & $5.0 \times 10^{5}$ \\
$\left|1^{\prime}\right\rangle \rightarrow|3\rangle$ & $\mathbf{5 . 6} \times \mathbf{1 0}^{-\mathbf{4}}$ & $\mathbf{3 . 3} \times \mathbf{1 0}^{\mathbf{4}}$ & $1.2 \times 10^{6}$ \\
$\left|1^{\prime}\right\rangle \rightarrow|4\rangle$ & $2.2 \times 10^{-8}$ & $\mathbf{2 . 3} \times \mathbf{1 0}^{\mathbf{4}}$ & $\mathbf{5 . 7} \times \mathbf{1 0}^{\mathbf{7}}$ \\
$\left|1^{\prime}\right\rangle \rightarrow|5\rangle$ & $1.9 \times 10^{-10}$ & $\mathbf{4 . 7} \times \mathbf{1 0}^{\mathbf{4}}$ & $\mathbf{3 . 5} \times \mathbf{1 0}^{\mathbf{7}}$ \\
\hline \hline
\end{tabular}

$100 \mathrm{~K}$. For each temperature, the peaks extracted from the FT are in excellent agreement with the relative value of the different scattering rates. It is finally worth stressing that the temperature variation of these rates is governed by the LO phonon population $n_{\mathrm{LO}}(T)$ and by the electron in-plane distribution. This is illustrated in the Fig. 4, where we consider, in a very crude approximation, that the absorption rate $\Gamma_{1^{\prime}, i}^{\mathrm{LO} \text { phonon-a }}$ towards the $i^{\text {th }}$ level is proportional to the fraction of mobile electrons in the $\left|1^{\prime}\right\rangle$ subband that can overcome the $\Delta E_{i, 1^{\prime}}$ energy difference by the absorption of one LO phonon:

$$
\begin{aligned}
\Gamma_{1^{\prime}, i}^{\mathrm{LO} \text { phonon-a }} & \propto n_{\mathrm{LO}}(T) \exp \left(-\frac{\Delta E_{i, 1^{\prime}}-\hbar \omega_{\mathrm{LO}}}{k_{\mathrm{B}} T}\right) \\
I_{\text {dark }}(T) & =I_{\text {dark }}(0)+a_{0} \sum_{i=2}^{5} \Gamma_{1^{\prime}, i}^{\mathrm{LO} \text { phonon-a }}(T) \mathrm{e}^{-2 \kappa_{i} \Delta z_{i, 1^{\prime}}},
\end{aligned}
$$

where $I_{\text {dark }}(0)$ and $a_{0}$ are fitting parameters, and the $T$ independent terms roughly account for the tunnel barrier to reach the final level $\left(\kappa_{i}=\sqrt{2 m^{*}\left(V_{0}-E_{i}\right) / \hbar^{2}}\right.$ and $\Delta z_{i, 1^{\prime}}$ the intermediate barrier width $(\approx 0,60,100$ and $100 \AA$ for $i=5,4,3$ and 2, respectively)). The line in Fig. 4 corresponds to $I_{\text {dark }}$ and is in agreement with experimental data. Concerning $I_{\text {dark }}(0)$, note that: (i) it may be due to assisted scattering processes involving $|1\rangle$ and $\left|1^{\prime}\right\rangle$, which are expected to be small (owing to the large spatial separation of the two states) and either independent (static disorder) or weakly dependent (acoustical phonons) on temperature; (ii) it is consistent with small current densities (typically in the $\mathrm{nAcm}^{-2}$ range) that have been reported in QWIPs with weakly coupled wells. ${ }^{28,29}$

To conclude this section, as well as the establishment of the different transitions involved in the dark current, their importance as a function of temperature is also demonstrated. At low temperature, only levels $|2\rangle$ and $|3\rangle$ contribute to $I_{\text {dark }}$ as it is schematized in Fig. 3(b). On the contrary, at higher temperatures, mainly levels $|4\rangle$ and $|5\rangle$ contribute to $I_{\text {dark }}$ (see Fig. 3(d)). Finally, an intermediate regime is highlighted which involves all the levels of the structure (see Fig. 3(c)). Measurements and calculations both confirm the intuitive idea that with increasing temperature, the dominating transitions involved in the dark equilibrium are more and more vertical in real space. In addition, this study highlights an important rule for the design of a high-performance QCD. Ideally the proba- 


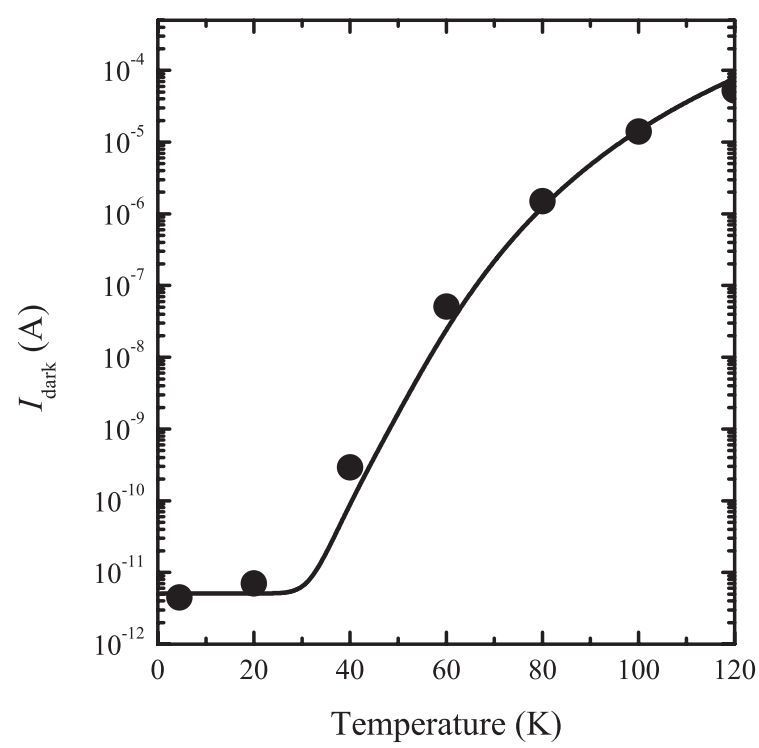

FIG. 4: Measured (symbols) and fitted (line) dark current at $V=$ $-0.1 \mathrm{~V}$ as a function of the temperature at $B=0 \mathrm{~T}$.

bilities of diagonal transitions $\left|1^{\prime}\right\rangle \rightarrow|i\rangle$ with $i=2,3,4$ have to be as low as possible. As written in Eq. (3), the intercascade current depends on $\Delta z_{i, 1^{\prime}}$ and $\Delta E_{i, 1^{\prime}}$. The former controls the overlap between the wavefunctions via the evanescent extension $\kappa_{i}$, and the latter gives the number of electrons in $\left|1^{\prime}\right\rangle$ that have sufficient energy to be promoted by LO phonon absorption to $|i\rangle$. For short wavelength structures with high optical energy such as the $8 \mu \mathrm{m}$ QCD, the cascade can be abrupt and benefit from efficient LO phonon emission without creating dark leakage. On the contrary, at longer wavelength, e.g. $14.3 \mu \mathrm{m}$, experiment reveals that the cascade has to have a flatter slope. This is the case in the studied QCD: the cascade is constituted of four QWs whereas only two would be enough to have two phonons stage. To avoid diagonal transitions, the levels of the cascade have to be spatially separated because the $\Delta E_{i, 1^{\prime}}$ have low energies.

\section{QCD UNDER ILLUMINATION}

Experimental setup used for magnetophotocurrent measurements is very similar to the one described in Sec. III: the QCD is mounted inside an insert at the center of a superconducting coil, $B$ being applied perpendicular to the growth axis. Light is emitted by a globar source from a Fourier transform infrared spectrometer and guided towards the sample which is back side illuminated. The experiment consists in measuring the current under illumination $I_{\text {light }}$ at zero voltage, the magnetic field being swept from 0 to $15 \mathrm{~T}$. The experiment has been performed at $T=20 \mathrm{~K}$, which corresponds to the temperature where the best detectivity of this QCD has been reported. ${ }^{8}$

The experimental curve is shown in Fig. 6(a). The photocurrent exhibits clear oscillations as a function of $B$ superposed on a general decreasing behaviour due to the magne-

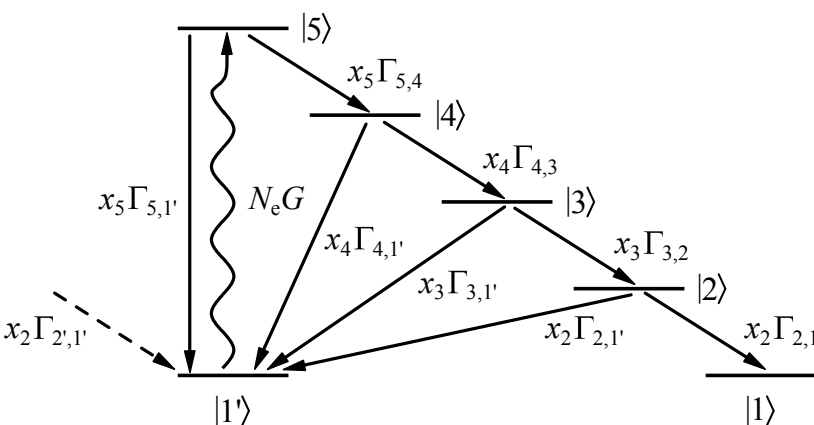

FIG. 5: Schematization of the QCD and scattering rates involved in the model of photocurrent. Electrons can only scatter between two consecutive levels $|i\rangle \rightarrow|i-1\rangle$ in the cascade and towards the ground state $|i\rangle \rightarrow\left|1^{\prime}\right\rangle$.

toresistance of the contacts. The arguments to understand the oscillatory part of the experimental data are based on the Landau quantization previously described in Sec. III. The dark current is well described by solving the electron balance of the intercascade transitions. As for the photocurrent, it results from a displacement of electrons after a photon absorption. This perturbation of the dark equilibrium is fruitfully described with a model that follows the photoelectrons while they go down the cascade. A complete description of the mechanisms involved in the photocurrent and a model describing it as a function of $B$ are presented in the following section.

\section{MODEL DESCRIBING PHOTOCURRENT}

In this section, we describe the model that explains the magnetophotocurrent oscillations in the QCD. The model of transport proposed is based on a rate equation approach within one single period. We assume electrons can only scatter between two consecutive levels $|i\rangle \rightarrow|i-1\rangle$ in the cascade and towards the ground state $|i\rangle \rightarrow\left|1^{\prime}\right\rangle$ as schematized in Fig. 5 . This model is a generalization of the previous one presented in Ref. 20. The sheet density $n_{i}$ of level $|i\rangle$ is $n_{i}=n_{i}^{\text {eq }}+x_{i}$, where $n_{i}^{\text {eq }}$ is the sheet density at equilibrium (no bias applied and without illumination) and $x_{i}$ the sheet density due to photon absorption. $x_{i}$ and $n_{i}$ follow

$$
\sum_{i=1}^{5} x_{i}=0 \quad \text { and } \quad \sum_{i=1}^{5} n_{i}=N_{\mathrm{e}}
$$

Under illumination, as illustrated in the Fig. 5, the rate equations relating $x_{i}$, the absorption efficiency coefficient $G-$ 
supposed constant — and their time derivatives read:

$$
\left\{\begin{array}{l}
\frac{\mathrm{d} x_{5}}{\mathrm{~d} t}=N_{\mathrm{e}} G-x_{5} \Gamma_{5} \\
\frac{\mathrm{d} x_{k}}{\mathrm{~d} t}=x_{k+1} \Gamma_{k+1, k}-x_{k} \Gamma_{k} \quad \text { with } k=2,3,4 \\
\frac{\mathrm{d} x_{1^{\prime}}}{\mathrm{d} t}=-N_{\mathrm{e}} G+\sum_{i=2}^{5} x_{i} \Gamma_{i, 1^{\prime}}
\end{array}\right.
$$

where $\forall i \in\{2, \ldots, 5\}, \Gamma_{i}=\Gamma_{i, 1^{\prime}}+\Gamma_{i, i-1}$ and

$$
\Gamma_{k, l}=\Gamma_{k, l}^{\mathrm{LO} \text { phonon-e }}+\Gamma_{k, l}^{\text {roughness }}+\Gamma_{k, l}^{\mathrm{impurities}}
$$

is the global scattering rate of an electron from level $|k\rangle$ to level $|l\rangle$ including LO-phonon emission, roughness and ionized impurities mechanisms. The current we are measuring in our macroscopic experimental setup is given by

$$
\frac{J}{q}=x_{2} \Gamma_{2,1},
$$

where $J$ is the current density and $q$ the charge of electron. In stationary state, solutions of Eqs. (5) combined with Eq. (7) leads to

$$
\frac{J}{q}=N_{\mathrm{e}} G \eta_{\mathrm{tot}}
$$

where

$$
\eta_{\mathrm{tot}}=\prod_{i=2}^{5} \eta_{i}=\frac{\Gamma_{5,4} \Gamma_{4,3} \Gamma_{3,2} \Gamma_{2,1}}{\Gamma_{5} \Gamma_{4} \Gamma_{3} \Gamma_{2}}
$$

with

$$
\forall i \in\{2, \ldots, 5\}, \eta_{i}=1-\frac{\Gamma_{i, 1^{\prime}}}{\Gamma_{i}}=\frac{\Gamma_{i, i-1}}{\Gamma_{i}}
$$

The $\eta_{i}$ represent the probability for an electron on level $|i\rangle$ to go down the cascade instead of going back on level $\left|1^{\prime}\right\rangle$. $\eta_{\text {tot }}$ represents the probability for an electron on level $\left|1^{\prime}\right\rangle$ to scatter on level $|1\rangle$, in other word this is the fraction of electrons that goes from one active well to the other one thanks to photon absorption. This coefficient is a good figure of merit to evaluate the efficiency of the QCD.

As mentioned in Ref. 20, three mechanisms are known to be dominant in midinfrared GaAs QCD - ionized impurities, LO-phonon and interface roughness. The calculated scattering rates of these different processes at $B=0 \mathrm{~T}, T=0 \mathrm{~K}$ and zero voltage are presented in Table II. LO-phonon scattering rate has been calculated following Ref. 30. For interface roughness, we used a Gaussian autocorrelation of the roughness with an average height of $\Delta=2.8 \AA$ and a correlation length of $\Lambda=60 \AA .^{20,31}$ For ionized impurities scattering, we used Ref. 20. Table II shows that the predominant mechanism for $\Gamma_{i, 1^{\prime}}$ and $\Gamma_{5,4}$ is ionized impurities scattering. This emphasizes the trade-off of the location of doping in the first quantum well, in agreement with previous studies. ${ }^{20}$ On the one hand doping is a limiting scattering because electrons on levels $|i\rangle$ scatter back to level $\left|1^{\prime}\right\rangle$. On the other hand, it enhances
TABLE II: Scattering rates in $\mathrm{s}^{-1}$ for different electronic scattering processes from level $|k\rangle$ to level $|l\rangle$ at $B=0 \mathrm{~T}$. The dominant mechanisms are in bold.

\begin{tabular}{cccc}
\hline \hline$|k\rangle \rightarrow|l\rangle$ & $\Gamma_{k, l}^{\text {LO phonon-e }}$ & $\Gamma_{k, l}^{\text {roughness }}$ & $\Gamma_{k, l}^{\text {impurities }}$ \\
\hline$|5\rangle \rightarrow\left|1^{\prime}\right\rangle$ & $1.6 \times 10^{12}$ & $2.4 \times 10^{12}$ & $\mathbf{2 . 0} \times \mathbf{1 0}^{\mathbf{1 3}}$ \\
$|4\rangle \rightarrow\left|1^{\prime}\right\rangle$ & $6.6 \times 10^{10}$ & $9.1 \times 10^{10}$ & $\mathbf{7 . 4} \times \mathbf{1 0}^{\mathbf{1 1}}$ \\
$|3\rangle \rightarrow\left|1^{\prime}\right\rangle$ & $8.2 \times 10^{8}$ & $7.5 \times 10^{8}$ & $\mathbf{8 . 4} \times \mathbf{1 0}^{\mathbf{9}}$ \\
$|2\rangle \rightarrow\left|1^{\prime}\right\rangle$ & $2.3 \times 10^{8}$ & $1.6 \times 10^{8}$ & $\mathbf{2 . 2} \times \mathbf{1 0}^{\mathbf{9}}$ \\
$|5\rangle \rightarrow|4\rangle$ & $6.1 \times 10^{4}$ & $5.7 \times 10^{11}$ & $\mathbf{2 . 7} \times \mathbf{1 0}^{\mathbf{1 3}}$ \\
$|4\rangle \rightarrow|3\rangle$ & $7.8 \times 10^{9}$ & $5.4 \times 10^{11}$ & $\mathbf{9 . 9} \times \mathbf{1 0}^{\mathbf{1 1}}$ \\
$|3\rangle \rightarrow|2\rangle$ & $2.3 \times 10^{0}$ & $8.2 \times 10^{12}$ & $\mathbf{1 . 7} \times \mathbf{1 0}^{\mathbf{1 3}}$ \\
$|2\rangle \rightarrow|1\rangle$ & $\mathbf{1 . 7} \times \mathbf{1 0}^{\mathbf{1 0}}$ & $\mathbf{1 . 7} \times \mathbf{1 0}^{\mathbf{1 0}}$ & $8.1 \times 10^{4}$ \\
\hline \hline
\end{tabular}

TABLE III: Extrapolated values of $\eta_{i}$ at $B=0 \mathrm{~T}$.

\begin{tabular}{ccccc}
\hline \hline$\eta_{i}$ & $\eta_{5}$ & $\eta_{4}$ & $\eta_{3}$ & $\eta_{2}$ \\
\hline$(\%)$ & 55 & 64 & 100 & 93 \\
\hline \hline
\end{tabular}

the scattering from $|5\rangle$ to $|4\rangle$, which improves the performance of the QCD. Besides, we notice that inside the cascade, impurities scattering is most of the time the dominant mechanism, prevailing over the LO-phonon mechanism, supposed to control the flow of electrons at the structure conception. Indeed the differences of energy involved in intercascade transitions are predominantly smaller than $\hbar \omega_{\text {LO }}$ making elastic scattering mechanisms dominant. This reinforces what we pointed out previously, ${ }^{20}$ that the design of the cascade has to fullfil a trade-off: a close cascade to efficiently extracts carriers and far enough to limit backscattering.

\section{ANALYSIS OF EXPERIMENTAL DATA UNDER ILLUMINATION}

In this section, we focus on the interpretation of photocurrent $I_{\text {light }}$ as a function of $B$ owing to Eqs. (8) and (9). To modelize $I_{\text {light }}$ we need to compute $\eta_{\text {tot }}$ as a function of $B$, i.e. calculate the scattering rates of the different elastic and inelastic mechanisms involved in the QCD. The first part of Eq. (9) shows that $\eta_{\text {tot }}$ is the product of 4 terms. It is thus valuable to compare $I_{\text {light }}$ with each one of these terms. Each $\eta_{i}$, for $i=5,4,3$ and 2 has been plotted in Fig. 6(b), (c), (d) and (e) respectively. According to Ref. 30, we considered a Gaussian broadening of the Landau levels with a width $\delta=4 \mathrm{meV}$, a value consistent with $\hbar \Gamma_{5,1^{\prime}}$ (see below).

The first interesting numbers are the values of $\eta_{i}$ obtained by extrapolation of the data under magnetic field at $B=0 \mathrm{~T}$. They are presented in Table III. As mentioned in Sec. V, the key point in QCD electronic transport is the transit of electrons from the upper level of the active region (level $|5\rangle$ ) to the cascade. In the present case, only $55 \%$ of photoabsorbed elec- 


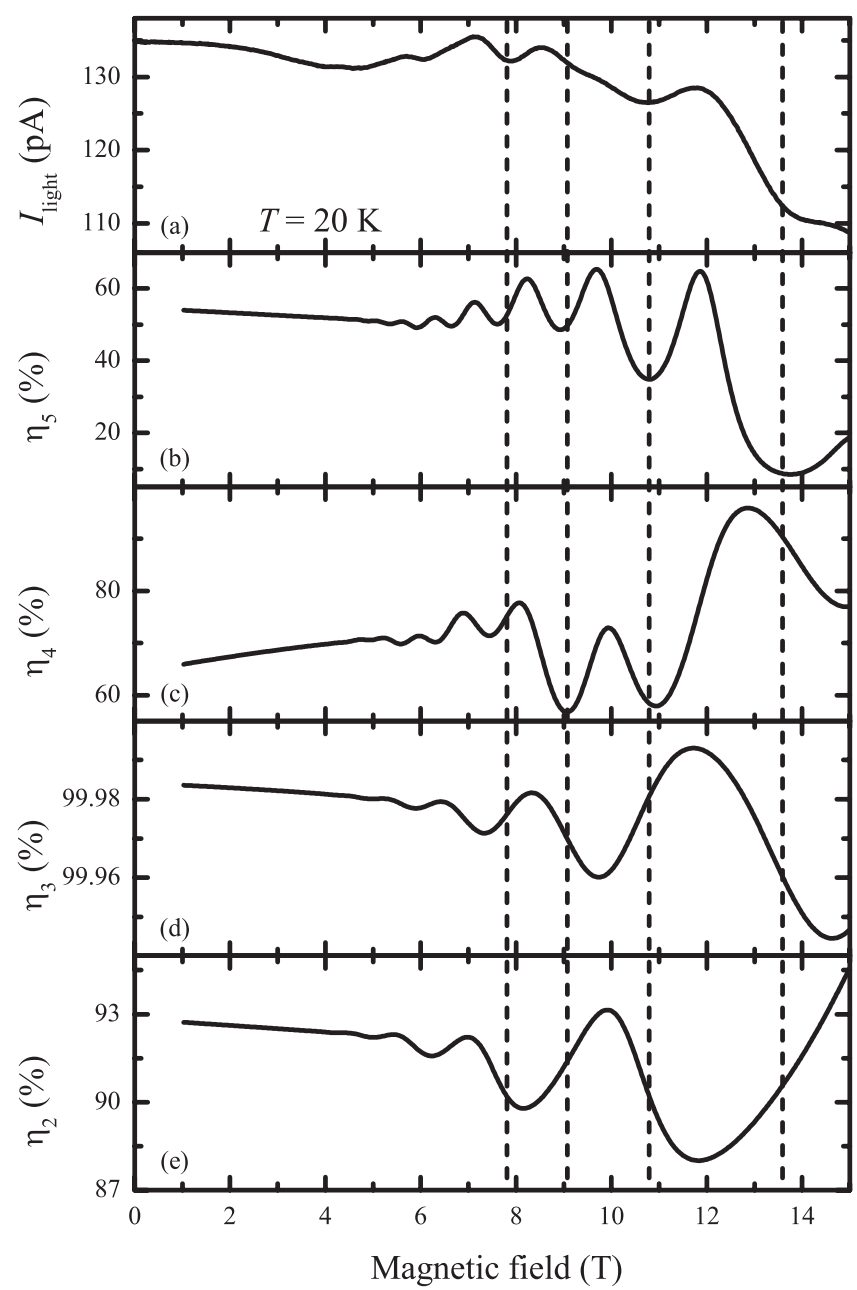

FIG. 6: (a) Experimental curve of photocurrent $I_{\text {light }}$ as a function of magnetic field. (b,c,d,e) Evolution of the factors (b) $\eta_{5}$, (c) $\eta_{4}$, (d) $\eta_{3}$ and (e) $\eta_{2}$ as a function of the magnetic field. These factors $\eta_{i}$ appear in the model describing the photocurrent as a function of $B$ in Eq. (10).

trons go from $|5\rangle$ to $|4\rangle$ and then $64 \%$ from $|4\rangle$ to $|3\rangle$. These fractions are a consequence of the electron-ionized impurities scattering that transfers backward a non-negligible part of electrons from level $|5\rangle$ to level $\left|1^{\prime}\right\rangle$. On the other hand, once engaged in the cascade, electrons flow with a high efficiency $(100 \%$ and $93 \%)$ thanks to all the mechanisms involved, as discussed in Sec. V. As a consequence, the oscillating behaviour in $I_{\text {light }}$ is governed by the $\eta_{i}$ that oscillates with the highest amplitude, $\eta_{5}$. This explains the good agreement of the minima positions between $I_{\text {light }}$ and $\eta_{5}$. On the opposite none of $\eta_{i=2,3,4}$ can account for oscillations in photocurrent.

The complete analysis is made by comparing $\eta_{\text {tot }}$ and $I_{\text {light }}$ as indicated by Eq. (8), $G$ and $N_{\mathrm{e}}$ being supposed constant. The experimental $I_{\text {light }}$ is shown in Fig. 7(a). The opposite of its second derivative $-\mathrm{d}^{2} I_{\text {light }} / \mathrm{d} B^{2}$ is plotted as a function of $B$ in Fig. 7(b). We plotted the opposite of the second derivative so that its maxima correspond to maxima of $I_{\text {light }}$. The coefficient $\eta_{\text {tot }}$ is presented in Fig. 7(c). It has been mentioned

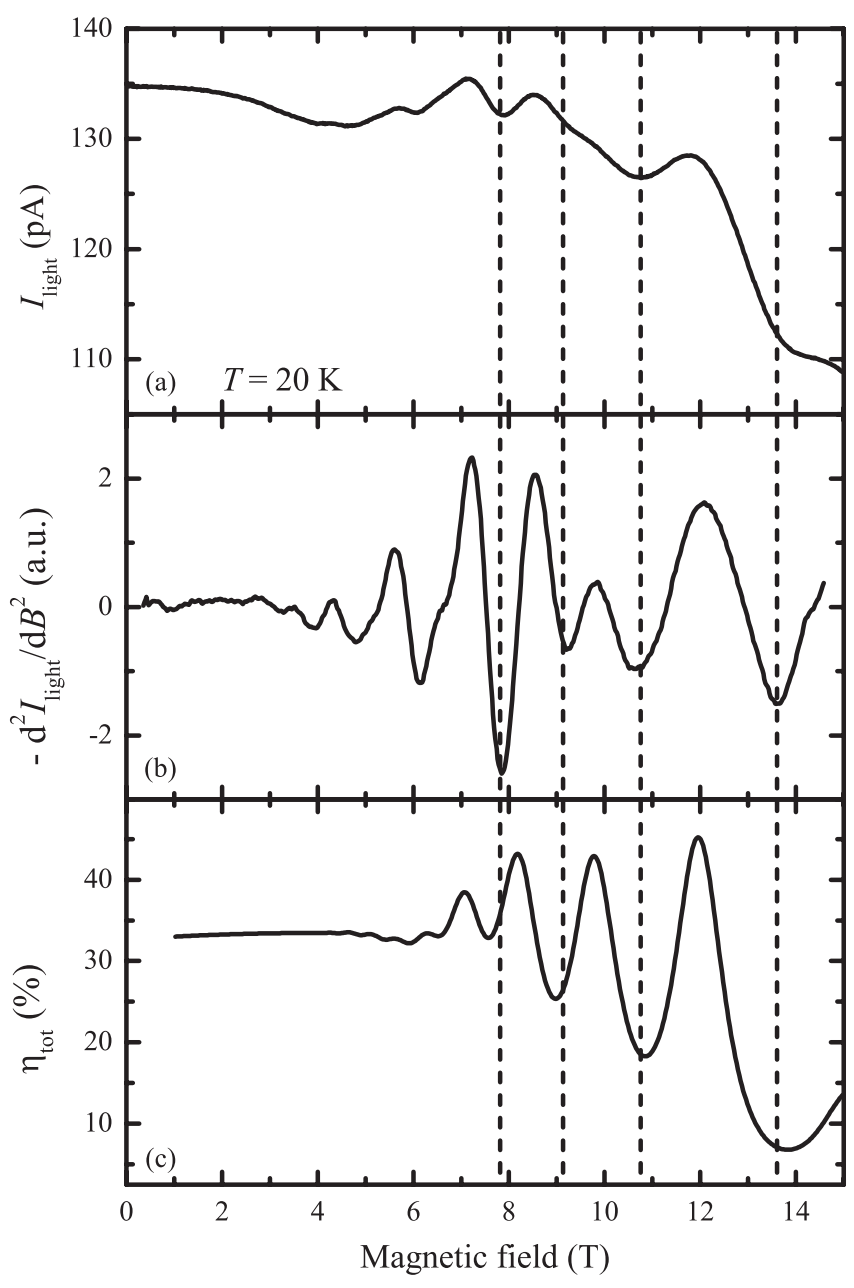

FIG. 7: (a) Experimental curve $I_{\text {light }}$ as a function of the magnetic field. (b) Opposite of the second derivative of $I_{\text {light }}$ as a function of $B$. (c) Evolution of $\eta_{\text {tot }}$ as a function of the magnetic field.

previously that the oscillating behaviour originates principaly from the ionized impurities scattering process involved in the electronic transfer from $|5\rangle$ to $\left|1^{\prime}\right\rangle$. This transfer leads to the minima in the photocurrent which fit well with $\eta_{\text {tot }}$. This can be clearly seen on the second derivative curve where minima and maxima are well pointed out. As explained above, $\eta_{5}$ is the main component of $\eta_{\text {tot }}$. This appears obviously comparing Fig. 6(b) and Fig. 7(c). The effect of $\eta_{4}, \eta_{3}$ and $\eta_{2}$ is to slowly modulate $\eta_{5}$ and to reduce the global proportion of electrons flowing from one active well to the next one. Under magnetic field, $\eta_{\text {tot }}$ oscillates between $7 \%$ and $45 \%$. By extrapolating the curve at $B=0 \mathrm{~T}$, one obtains $\eta_{\text {tot }}=33 \%$, a value that should be increased to improve the detector performance. This analysis shows the importance during the initial design of the band structure to drastically optimize the extraction from the level $|5\rangle$ to the cascade.

Finally, we also developed a more complex model, where electron could scatter not only between two consecutive levels $|i\rangle \rightarrow|i-1\rangle$ but also between $|i\rangle \rightarrow|i-2\rangle$. This model could better take into account non elastic scattering, particularly the LO-phonon scattering, which becomes important 
when the difference of energy between two levels is higher than $\hbar \omega_{\mathrm{LO}}=36 \mathrm{meV}$. However, one obtains that the general behaviour of $\eta_{\text {tot }}$ remains essentially unchanged: the quantitative change is inferior to $7 \%$ (extrapolation at $B=0 \mathrm{~T}$ gives $35 \%)$.

Resonant tunneling has not been taken into account in this calculation. Looking at the small overlap between wavefunctions $|5\rangle$ and $|4\rangle$ on Fig. 1, it is not expected to have a significant impact in the overall calculation. We have shown already that an elastic mechanism dominates the extraction inside the cascade. Resonant tunneling is more dominant in the $|3\rangle \rightarrow|2\rangle$ transition, but calculation of the wavefunctions $|3\rangle$ and $|2\rangle$ in the symetric/antisymetric basis already accounts for it. $^{32}$

\section{CONCLUSION}

In conclusion, magnetotransport experiments have been performed in a complex multi quantum well structure as a VLWIR QCD. The current in this structure, both in dark condition and under illumination, is the combination of several electronic paths due to different scattering mechanisms difficult to analyse together. In these conditions, the magnetic field has proven to be a powerful tool to highlight separately the different channels involved in the current.

Dark current study has revealed three regimes of transport. At low temperature $(T \leqslant 20 \mathrm{~K})$, low energy transitions are dominant whereas at high temperature $(T \geqslant 80 \mathrm{~K})$, higher energy transitions are involved thanks to thermal activation.
Influence of temperature plays a key role because in the VLWIR range, the difference of energy between the lower and the upper level of the optically active QW is smaller than in long wave infrared. These results are in agreement with the best performances observed and used for the working operation of this QCD $(T \sim 20 \mathrm{~K})$.

Under illumination, a model has been developed to describe the oscillating part of the magnetophotocurrent. This model has the advantage to include all the levels of the structure, which was not the case in a previous study. ${ }^{20}$ From a fundamental point of view, it reveals the subtle entanglement of scattering mechanisms that occurs in quantum well structures. The transport properties are the result of multiple trade-offs : the cascade has to be efficient, i.e. have a good extraction but should be distant enough from level $\left|1^{\prime}\right\rangle$; ionized impurities promote extraction but enhance the backscattering from the cascade to $\left|1^{\prime}\right\rangle$; transport in the cascade was thought to be principally governed by LO phonon during the design conception of the QCD but the different scattering mechanisms contribute actually. For applications, this study gives the key points to optimize the design of the new structures for the next generation of VLWIR QCD.

\section{ACKNOWLEDGMENTS}

The Laboratoire Pierre Aigrain is a "Unité Mixte de Recherche" (UMR 8551) between École Normale Supérieure, the CNRS, the University Pierre et Marie Curie (Paris 6) and the University Paris Diderot (Paris 7).
* Present address: SOFRADIR, BP 21 - 38113 Veurey-Voroize France

$\dagger$ Electronic address: louis-anne.devaulchier@lpa. ens.fr

${ }^{1}$ L. Gendron, M. Carras, A. Huynh, V. Ortiz, C. Koeniguer, and V. Berger, Appl. Phys. Lett. 85, 2824 (2004).

2 A. Vardi, G. Bahir, F. Guillot, C. Bougerol, E. Monroy, S. E. Schacham, M. Tchernycheva, and F. H. Julien, Appl. Phys. Lett. 92, 011112 (2008).

${ }^{3}$ M. Graf, G. Scalari, D. Hofstetter, J. Faist, H. Beere, E. Linfield, D. Ritchie, and G. Davies, Appl. Phys. Lett. 84, 475 (2004).

${ }^{4}$ G. Scalari, M. Graf, D. Hofstetter, J. Faist, H. Beere, and D. Ritchie, Semicond. Sci. Technol. 21, 1743 (2006).

${ }^{5}$ L. Gendron, C. Koeniguer, V. Berger, and X. Marcadet, Appl. Phys. Lett. 86, 121116 (2005).

${ }^{6}$ M. Graf, N. Hoyler, M. Giovannini, J. Faist, and D. Hofstetter, Appl. Phys. Lett. 88, 241118 (2006).

${ }^{7}$ F. R. Giorgetta, E. Baumann, M. Graf, L. Ajili, N. Hoyler, M. Giovannini, J. Faist, and D. Hofstetter, Appl. Phys. Lett. 90, 231111 (2007).

8 A. Buffaz, M. Carras, L. Doyennette, A. Nedelcu, X. Marcadet, and V. Berger, Appl. Phys. Lett. 96, 172101 (2010).

${ }^{9}$ G. Sarusi, S. D. Gunapala, J. S. Park, and B. F Levine, J. Appl. Phys. 76, 6001 (1994)

10 A. Nedelcu, V. Guriaux, A. Bazin, L. Dua, A. Berurier, E. Costard, P. Bois, and X. Marcadet, Infrared Phys. Technol. 52, 412 (2009).

11 P.-O. Lagage, C. Doucet, E. Pantin, E. Habart, G. Duchne, F.
Ménard, C. Pinte, S. Charnoz, and J.-W. Pel, Science, 314, 621 (2006).

${ }^{12}$ H. Schneider and H. C. Liu, Quantum Well Infrared Photodetectors, Physics and applications (Springer, Heidelberg, 2007).

${ }^{13}$ S. Blaser, M. Rochat, M. Beck, D. Hofstetter, and J. Faist, Appl. Phys. Lett. 81, 67 (2002).

14 D. Smirnov, O. Drachenko, J. Leotin, H. Page, C. Becker, C. Sirtori, V. Apalkov, and T. Chakraborty, Phys. Rev. B 66, 125317 (2002).

15 G. Scalari, C. Walther, L. Sirigu, M. L. Sadowski, H. Beere, D. Ritchie, N. Hoyler, M. Giovannini, and J. Faist, Phys. Rev. B 76, 115305 (2007).

16 A. Leuliet, A. Vasanelli, A. Wade, G. Fedorov, D. Smirnov, G. Bastard, and C. Sirtori, Phys. Rev. B 73, 085311 (2006).

${ }^{17}$ N. Péré-Laperne, L.-A. de Vaulchier, Y. Guldner, G. Bastard, G. Scalari, M. Giovannini, J. Faist, A. Vasanelli, S. Dhillon, and C. Sirtori, Appl. Phys. Lett. 91, 062102 (2007).

18 F.-R. Jasnot, L.-A. de Vaulchier, Y. Guldner, G. Bastard, A. Vasanelli, C. Manquest, C. Sirtori, M. Beck, and J. Faist, Appl. Phys. Lett. 100, 102103 (2012).

19 A. Gomez, N. Péré-Laperne, L.-A. de Vaulchier, C. Koeniguer, A. Vasanelli, A. Nedelcu, X. Marcadet, Y. Guldner, and V. Berger, Phys. Rev. B 77, 085307 (2008).

${ }^{20}$ F.-R. Jasnot, N. Péré-Laperne, L.-A. de Vaulchier, Y. Guldner, F. Carosella, R. Ferreira, A. Buffaz, L. Doyennette, V. Berger, M. Carras, and X. Marcadet, Phys. Rev. B 82, 125447 (2010).

${ }^{21}$ B. S. Williams, Nature Photon. 3, 732 (2009). 
${ }^{22}$ A. Delga, M. Carras, L. Doyennette, V. Trinité, A. Nedelcu, and V. Berger, Appl. Phys. Lett. 99, 252106 (2011).

23 A. Delga, M. Carras, V. Trinité, V. Guériaux, L. Doyennette, A. Nedelcu, H. Schneider, and V. Berger, Phys. Rev. B 85, 245414 (2012).

${ }^{24}$ H.M. Swartjes, A.G.M. Jansen and P. Wyder, Phys. Rev. B 38, 8114 (1988)

${ }^{25}$ C. Koeniguer, G. Dubois, A. Gomez, and V. Berger, Phys. Rev. B 74, 235325 (2006).

26 A. Buffaz, A. Gomez, M. Carras, L. Doyennette, and V. Berger, Phys. Rev. B 81, 075304 (2010).
${ }^{27}$ R. Ferreira and G. Bastard, Phys. Rev. B 40, 1074 (1989).

28 A. Gomez, V. Berger, N. Péré-Laperne, and L.-A. de Vaulchier, Appl. Phys. Lett. 92, 202110 (2008).

${ }^{29}$ F. Castellano, F. Rossi, J. Faist, E. Lhuillier, and V. Berger, Phys. Rev. B 79, 205304 (2009).

${ }^{30}$ C. Becker, A. Vasanelli, C. Sirtori, and G. Bastard, Phys. Rev. B 69, 115328 (2004)

${ }^{31}$ H. Sakaki, T. Noda, K. Hirakawa, M. Tanaka, and T. Matsusue, Appl. Phys. Lett. 51, 1934 (1987).

${ }^{32}$ H. Callebaut and Q. Hu, J. Appl. Phys. 98, 104505 (2005). 